\title{
The Implementation of Load Forecasting Platform Based on Cloud Computing
}

\author{
Li Weijun ${ }^{1}$, Pang Yanjun ${ }^{1}$, Wang Qinghao ${ }^{1}$, Liu Bo ${ }^{1}$, Wang Chuang ${ }^{1}$, Liu \\ Zhitong $^{2}$, Hao Guanqiao ${ }^{1}$, Zhao Chuanzong ${ }^{1}$, Xie Xinnan $^{1}, \mathrm{Xu} \mathrm{Mei}^{1}$ \\ ${ }^{1}$ Fushun Power Supply Company, Liaoning Electric Power Company Limited, State Grid, China, \\ ${ }^{2}$ Shenyang Power Supply Company, Liaoning Electric Power Company Limited, State Grid, China, \\ sptlove51@126.com
}

Keywords: cloud computing; smart grid; grid load forecasting platform

\begin{abstract}
As the rapid development of China's economy construction and scientific technology, the grid carrying load becomes higher and higher, and how to realize correct grid load forecasting technique becomes a current new smart grid studying hot spot. Without an exact calculation for the load, it can cause that the grid goes wrong in actual operation easily. Due to development and diffusion of cloud computing technology, it can realize load forecasting platform of Fushun smart grid by cloud computing technology at present. The thesis focuses on Fushun grid load forecasting, utilizes cloud computing technology to achieve implementation effects of grid load forecasting platform, and analyses and studies characters, main frame, primary technology, facing problems, related applications of Fushun grid load forecasting.
\end{abstract}

\section{Introduction}

It is very important for grid load forecasting platform to operate normally in Fushun. As some factories and mines bankruptcy, transfer, and new type enterprises develop, Fushun is old industrial city, if there is no perfect grid load forecasting platform, there is no perfect to forecast forthcoming load values accordingly. Once the load becomes higher, it will be great harm to grid, even leads to power grid accidents, and causes significant implications to people's lives and property in Fushun. The traditional grid load forecasting platform can't adapt to high load, big data current situation, for the same reason, there is important significance for economy construction and people's lives to achieve load forecasting platform of new smart grid. With the development of cloud computing technology, it will be applied to forecasting process of grid load. From characters of cloud computing and grid load forecasting platform frame, the thesis mainly discusses the implementation method of new smart grid load forecasting platform based on cloud computing.

\section{Implementation of cloud computing technology and smart grid load forecasting}

\section{Cloud computing technology overview}

Cloud computing is commercial large distributive computing technology, through the current network users break down the large computing program into several smaller subroutine automatically, hand the larger system that is composed of several servers, and get processed results by searching, calculating, analyzing. You can also reduce interaction of service providers effectively, and save much time to manage. It will play more and more important role in life, manufacture and commercial activity in future, and cause structure of internet to make fundamental change. So cloud computing technology has been paid close extensive attention in modern large internet companies, such as Alibaba, Baidu and so on. These famous firms are racing to study cloud computing services. This technology is mainly applied in the internet, scientific computer and web, and related studying is further developing.

\section{Characters of Fushun smart grid load forecasting platform}

During the traditional grid load forecasting using, there is no enough information to master in the forecasting process in general and the information degree is lower. It needs using information technology to solve this problem. Fushun smart grid has possessed "Sihua" characters at present, 
and it mainly refers to the "information, automation, digitization, interacting". During achieving of Fushun smart grid, involving power generation, transmission, substation, distribution, consumption and dispatch of six links. As to the six links, the new requirements are proposed to achieving of Fushun smart grid forecasting platform. During the load forecasting, one of the first issues newlyweds face is that analyzing and forecasting systems are more, the characteristics which every system possesses are different.

\section{Six features of forecasting platform}

During achieving of the actual power grid load forecasting platform, according to the shortage of the original system, it needs the following six features for the smart grid load forecasting platform to possess.

(1) Forecasting more objects

The process of using power energy has been different from the original process in Fushun, so grid load forecasting is from previous bus load forecasting and system load forecasting to a full range forecasting of "point-line-surface-body". That is to say, it is important to forecast bus load, area load, user load and the whole network load accordingly.

(2) Forecasting a variety of factors

In the smart grid environment, it needs to think about not only social factors and meteorological factors but also power generation grid plan, load management way and the influence of peak load shifting under the condition of energy storage. In this situation, the normal operating can achieve smart grid load forecasting.

(3) Finding the specific reasons of energy consumption

Smart grid load forecasting can find how power is consumed, only you finding out the reason of power consumption, you achieve the load forecasting of Fushun power grid.

(4) Forecasting high accuracy

In the process of traditional grid operation, information flow is often only one-way information, but in the process of using smart grid, power and information are two-way, at the same time the exchange of information is faster, the frequency of forecasting is also higher. When you achieve this function, you will master the law of load change accurately and make the accuracy of load forecasting higher.

(5) The better adaptability

According to the different objects, smart grid load forecasting select the corresponding model, the parameters should change with the changing of the object, the inflection point and extreme point appear at the time and size to have higher sensitivity.

(6) Intelligent information base

On the basis of traditional load forecasting, load forecasting should be as fine as possible, and establish the information base of intelligent management, load characteristic analysis library and influencing factors information database. In addition, in order to ensure the vitality of intelligent information database, load forecasting should also strengthen the contact with the public service platform. Grid load forecasting platform of Fushun power supply company has possessed above six features now, so implementing cloud computing is possible in Fushun grid load forecasting platform.

\section{The main framework of Fushun smart grid load forecasting}

In the process of achieving the smart grid, it needs to analyze different problems, use comprehensive knowledge and adaptive methods to forecast, and get better results.

From the forecasting process, the main framework of Fushun grid load forecasting includes the following steps: (1) Screening sample and dealing with initial abnormal data; (2) Selecting intelligent forecasting model in the middle; (3) Modifying forecasting results at last.

From the aspect of power generation and consumption, the main framework of Fushun grid load forecasting includes: (1) Establishing relationship between the total power load and influencing factors; (2) Establishing the relationship between the influencing factors with uncontrolled load; (3) According to the "unified dispatch load / net + uncontrolled load = total power load" relation, 
analyze changing law of unified dispatch load/ net. Figuratively speaking, three-dimensional structure of smart grid load forecasting is shown in Fig.1.

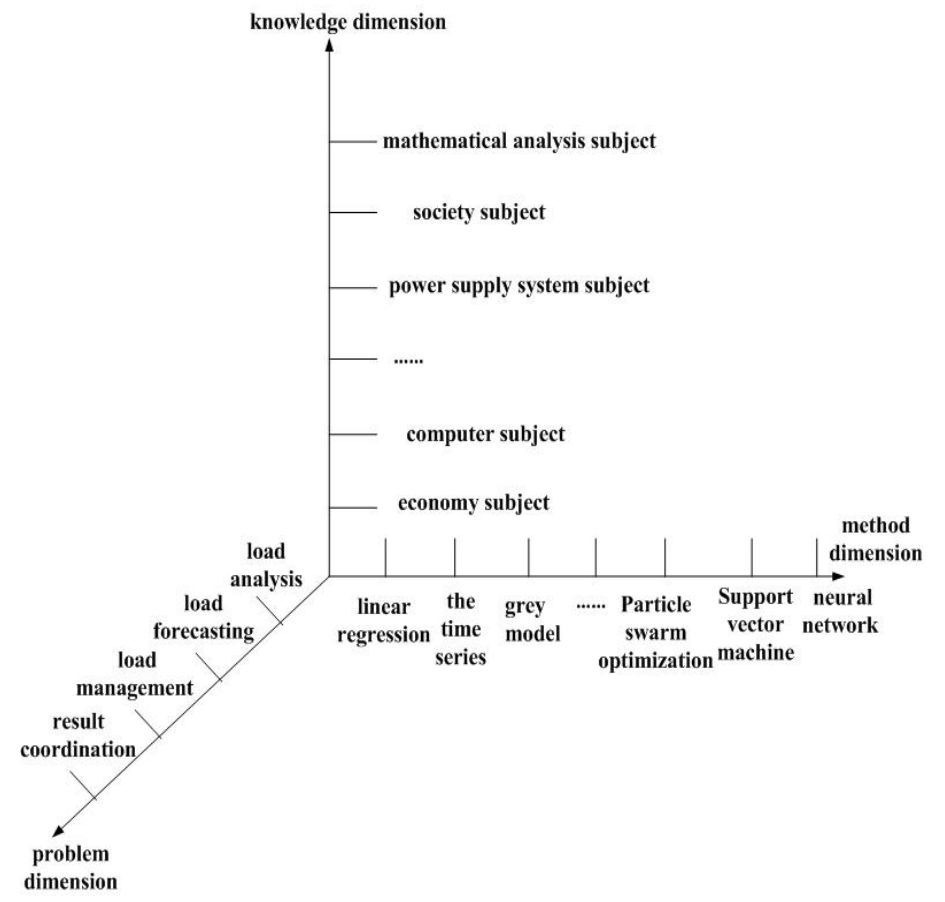

Fig.1 three-dimensional structure of smart grid load forecasting

It can be seen from the three-dimensional structure of load forecasting, if you need to achieve smart grid load forecasting system, the prediction system can be used to study the interaction of various factors in the smart grid environment, and the power consumption can be used to forecast the load of various electrical appliances with interactive features.

\section{Private cloud platform of Fushun power supply company}

Cloud computing is developing rapidly, deployment model with cloud computing can involve public cloud, private cloud, community cloud, hybrid cloud. Service quality and data security of private cloud is the best in the part, it has the advantages of high efficiency and integration of computing resources, someone uses system internal network to build power private cloud in the system. After building power private cloud, it can effectively improve the processing power of grid data and the interactive ability of multiple systems, the ability to collect, analyze, integrate and mine data in the whole power system is improved, build a low cost information network of power system, achieve the resource access and data storage to control their functions, it has the function which public cloud platform doesn't have. China's power grid uses standard for unified scheduling and hierarchical management to construct in the framework of the power dispatching private cloud, so dispatching mechanism of power system is divided into county dispatching, area dispatching, network dispatching and country network dispatching. Collecting data and monitoring management need to have a certain storage resources and processor resources at each level of the scheduling system. Due to achieving of power cloud, you can't change the physical distribution of existing computers; also need to allow the private cloud can use the physical architecture of existing power system at large, and put the physical architecture to distribute as tasks. In the actual case, there is the following dispatching architecture, it is shown in Fig.2. 


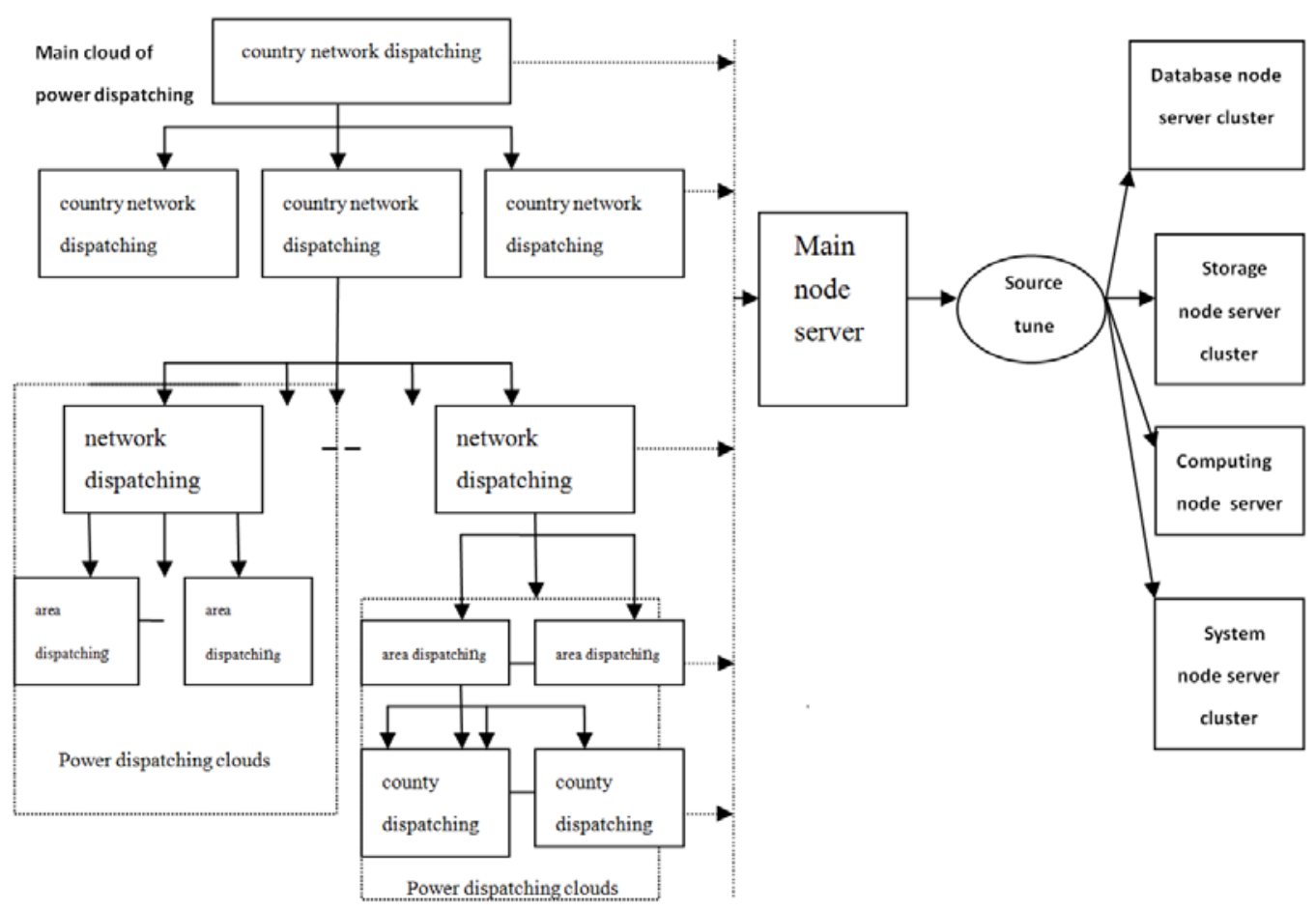

Fig.2 dispatching architecture

Using cloud technology achieves grid load forecasting platform, main principle is based on users’ specific needs. Specific load forecasting process is shown in Fig.3.

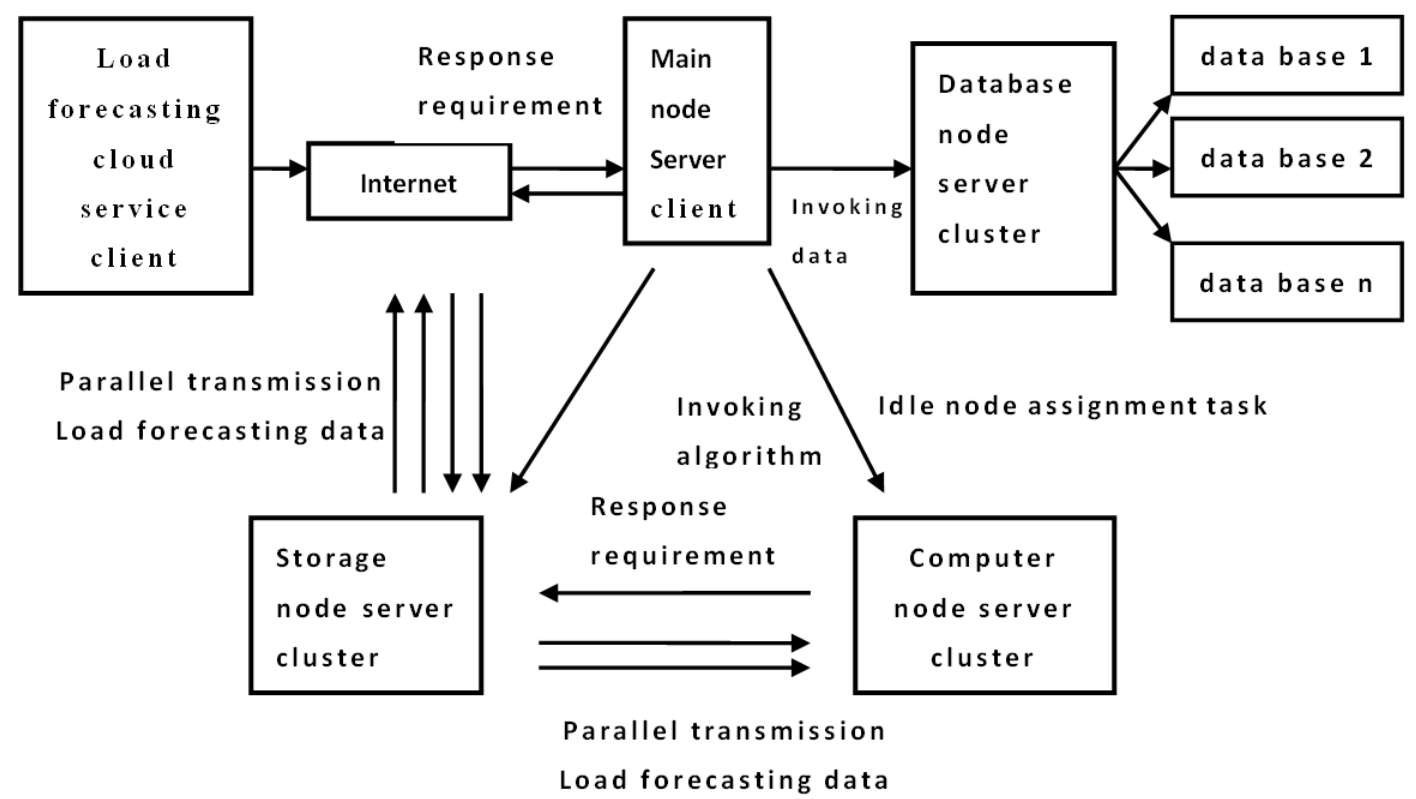

Fig.3 specific load forecasting process

In actual operation, service client requests firstly, and transfers the sent demand to the main node server. It obtains data from database node server, after obtaining data, then calls forecasting algorithm, and distributes resources to computing node server. Arrange different nodes and use fine process to analyze load characteristic, deal with data, ensure the parameters of the model and influence factors; Relevant data is required to be obtained from the storage node server at last, send to main server, analysis and forecasting results are sent to the user together.

\section{Conclusion}

Demand for power is huge in Fushun, while demand for load is variability, so it is very important for grid load forecasting. In such a big environment, it is urgent to study the new smart 
grid load forecasting platform. Because cloud computing technology possesses high reliability, virtualization, large scale, versatility and high sensitivity advantages, it is adapt to the actual needs of modern smart grid. Cloud computing utilizes a wide range of resources features, shares the operation data of the regional power network, and supports the time domain simulation of the whole power grid integration, thus improves the stability analysis level of the whole power grid greatly. So the introduction of cloud computing technology can greatly improve the smart grid load forecasting platform.

\section{References}

[1] Guoliang Zhou, Guilan Wang, Jia Ge. Short term power load forecasting technology based on cloud computing [J]. Electric power information, 2012, 10(3): 111-114.

[2] Jianbiao Cai. Research on smart grid load forecasting platform based on cloud computing[D]. Hunan University. 2013.

[3] Weiming Zhan. Research on parallel algorithm of generation scheduling in cloud computing environment[D]. North China Electric Power University,2013.

[4] Yanliu Cheng. Research on data mining of smart grid based on Cloud Computing[D]. North China Electric Power University,2013.

[5] Baoyi Wang, Shuo Zhao, Shaomin Zhang. Distributed load forecasting algorithm based on cloud computing and extreme learning machine[J]. Grid technology, 2014(02): 526-531. 